\title{
Synthesis and photoluminescence property of silicon carbide nanowires thin film by HF-PECVD system
}

\author{
ZHANG ENLEI*, WANG GUOSHENG, LONG XIAOZHU and WANG ZHUMIN \\ College of Chemical Engineering, Shenyang University of Chemical Technology, Shenyang 110142, \\ People's Republic of China
}

MS received 1 July 2013; revised 2 September 2013

\begin{abstract}
A sample and scalable synthetic strategy was developed for the fabrication of nanocrystalline SiC (nc-SiC) thin film. Thin sheet of nanocrystalline diamond was deposited on Si substrate by hot filamentassisted plasma-enhanced chemical vapour deposition system (HF-PECVD). Further, the resulting carbonbased sheet was heated at $1200{ }^{\circ} \mathrm{C}$ to allow a solid state reaction between $\mathrm{C}$ and $\mathrm{Si}$ substrate to form the SiC thin films. The synthesized films mainly consist of $\beta$-SiC nanowires with diameters of about $50 \mathrm{~nm}$ and tens of micrometers long. The nanowires axes lie along the $\left[\begin{array}{lll}1 & 1 & 1\end{array}\right]$ direction and possess a high density of planar defects. The $\beta$-SiC nanowires thin films exhibit the strong photoluminescence (PL) peak at a wavelength of $400 \mathrm{~nm}$, which is significantly shifted to the blue compared with the reported PL results of SiC materials. The blue shift may be ascribed to morphology, quantum size confinement effects of the nanomaterials and abundant structure defects that existed in the nanowires.
\end{abstract}

Keywords. SiC nanowires; nanocrystalline diamond; crystal growth; photoluminescence.

\section{Introduction}

SiC has been widely recognized as a versatile material with tremendous industrial applications because of its unique mechanical, electrical and thermal properties. Due to the wide band gap property, $\mathrm{SiC}$ is becoming a promising candidate for short wavelength light emitters, detecting devices and biosensors (Pauschitz et al 2000; Borowiak et al 2005; Fan et al 2006; Chen et al 2008). Generally, bulk SiC is not a good light applicable source to nano-optoelectronic integration on account of its indirect band gap (Fang et al 2012; Fernandez et al 2012; Guruvenket et al 2012). But various SiC nanostructures possess excellent photoluminescence (PL) property. Many investigators have focused their attention on the preparation and optical properties of $\mathrm{SiC}$ nanomaterial. They believe that the optical emission at short wavelength can be achieved through a combination of the wide band gap property and quantum confinement effect of nanocrystalline SiC (nc-SiC) (Huang and Hong 2006; Li et al 2007; Huong et al 2012). Hence nc-SiC thin films have attracted much attention in the recent years because of their potential applications in many kinds of optoelectronic devices, such as solar cells, image sensors and photodiodes (Pham 2001; Paul 2008; Lien et al 2011). However, only several literatures on PL performances of $\mathrm{nc}-\mathrm{SiC}$ thin film have been reported so far.

*Author for correspondence (zhangel1979@126.com)
SiC nanomaterials have been successfully synthesized using a number of methods including decomposition of organic silicon compounds, high temperature reactions between carbon nanotubes and $\mathrm{SiO}$, arc-discharge processes, hot filament chemical vapour deposition and lowtemperature, atmospheric microplasma deposition (Zhou et al 2000; Xu et al 2005; Wang 2007; Qamar et al 2011; Vaghri et al 2011). In addition, these techniques are very complex, slow and harmful because of the use of the highly toxic precursor, silane. Moreover, these processes are mostly based on the use of metal catalysts such as gold, nickel, aluminum and metal oxide, which are often undesirable in the final product due to contamination of metals affecting the performance of the electronic device.

In this paper, we report on a novel method for growing $\mathrm{SiC}$ nanowires thin film by hot filament-assisted plasma-enhanced chemical vapour deposition (HFPECVD) system. The results have shown that these nanowires have a single-crystalline $\mathrm{SiC}$ core and a thin amorphous $\mathrm{SiO}_{2}$ shell. The nc-SiC thin film shows a strong light emission centred at $400 \mathrm{~nm}$ at room temperature, which is significantly shifted to the blue compared with the reported several luminescence results of $\mathrm{SiC}$ nanowires and nanocables.

\section{Experimental}

The synthesized procedure of thin films consisting SiC nanowires were deposited using a double bias-voltage-assisted 
HF-PECVD system. First, silicon substrates were treated with piranha solution, $\mathrm{H}_{2} \mathrm{SO}_{4} / \mathrm{H}_{2} \mathrm{O}_{2}$ for $20 \mathrm{~min}$. Second, the substrates were washed vigorously by flushing water for several minutes and dried under an $\mathrm{N}_{2}$ flux. Finally, surface patterning of the substrate was achieved by pure Ar plasma (30 sccm Ar, $150 \mathrm{~W}$, substrate bias voltage $-200 \mathrm{~V}, 10 \mathrm{~s}$ ) before the deposition.

The deposition of thin films was carried out in the two bias-voltage HE-PECVD system. The base pressure was $7.5 \times 10^{-3} \mathrm{~Pa}$. By adjusting the flow rate of $\mathrm{CH}_{4}$, the working pressure was maintained at $10 \mathrm{~Pa}$. $\mathrm{CH}_{4}$ was used as the precursor with $60 \mathrm{sccm}$. The deposition time was $1 \mathrm{~h}$. The substrate bias voltage was $-120 \mathrm{~V}$ and the grid bias voltage was $100 \mathrm{~V}$. The current of hot filament was adjusted to $60 \mathrm{~A}$ in order to achieve a temperature of $1200{ }^{\circ} \mathrm{C}$, and the radio-frequency power was $80 \mathrm{~W}$. After the experiment, a slightly green thin film was formed on the surface of the substrate.

The morphology and crystal lattice of the samples were observed by field-emission transmission electron microscopy (TEM, JEOJ JSM-5600LV), high-resolution transmission electron microscopy (HR-TEM, JEOL JEM3010) and atomic force microscopy (AFM, AJ-III). The samples were carried out by AFM analysis in tapping mode. The topography of all the samples for a scanned area of $5 \times 5 \mu \mathrm{m}$ were evaluated for a set point of $10 \mathrm{nA}$ and a scan rate of $10 \mathrm{~mm} / \mathrm{s}$. XRD of the as-deposited thin film was included. The crystalline structure was analysed by X-ray diffraction (XRD, Semens D5000). The possible chemical composition of as-grown products was investigated by energy-dispersive X-ray spectroscopy (EDS) attached to the TEM. Raman spectroscopy with LabRa 010 was used to study the bonding characteristics. PL (PL, Hitatch F-4500) spectroscopy measurement was performed with xenon lamp under $354 \mathrm{~nm}$ as the excitation source at room temperature.

\section{Results and discussion}

The surface morphologies of the nanocrystalline diamond (NCD) film obtained on the Si substrate were analysed by SEM and AFM. It can be observed from figure 1(a) that the NCD films were deposited on the Si substrate. The thin films are composed of carbon nanoparticles, and the carbon nanoparitcles are well distributed and their sizes are uniform, indicating that NCD films are homogeneously formed on the substrates. There are no obvious defects in the film, such as porosity. Individual nanoparticles are clearly observed on the surface, only with certain aggregations that will not affect the film continuity. AFM image of the film deposited on the Si substrate displayed in figure 1(b). It shows that NCD films with root mean square roughness around $10 \mathrm{~nm}$ and an average grain size of $40 \mathrm{~nm}$ were obtained. It is further revealed that the particles are densely laid so as there are smooth surfaces.
Raman spectroscopy is such a prevailing and nondestructive technique to characterize the structural properties of carbon coating and their quality. The Raman spectra of as grown NCDs on the Si substrate show the three important peaks, as shown in figure 2. The peaks at
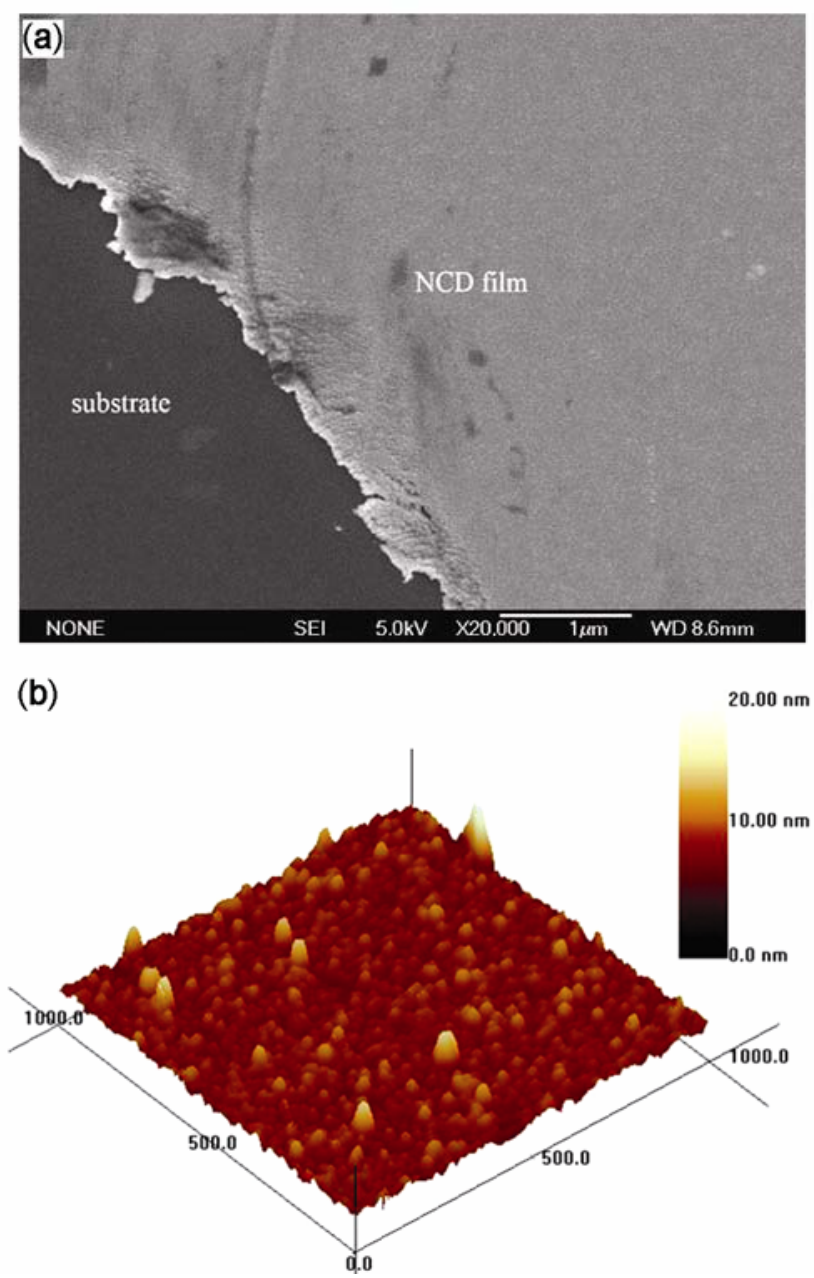

Figure 1. Morphology of NCD film deposited on Si Substrate. (a) SEM and (b) AFM.

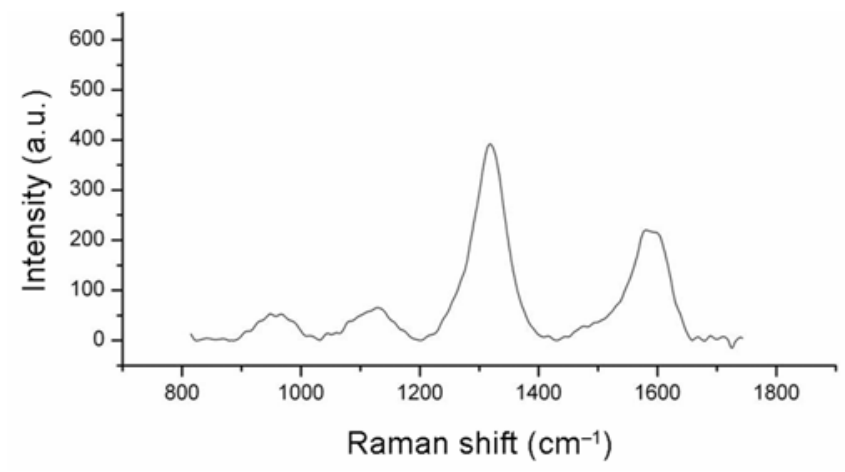

Figure 2. Raman spectrum of NCD film deposited on the Si substrate. 
1388 and $1571 \mathrm{~cm}^{-1}$ are related to D and G bands (Paul 2008; Hsu et al 2010; Vaghri et al 2011; Huang et al 2012). Whereas the D band for the NCD deposited on the Si substrate appeared at a low wavenumber at $1345 \mathrm{~cm}^{-1}$, also the $G$ peak position shifted to a lower wavenumber at $1517 \mathrm{~cm}^{-1}$. Raman spectroscopy shows clearly a broad peak around $1150 \mathrm{~cm}^{-1}$, characteristic of nanocrystalline diamond (Huang and Hong 2006). However, no SiC peaks were observed. At relatively low temperature (about $1000^{\circ} \mathrm{C}$ ), no reaction between NCD film and Si substrate took place, so no $\mathrm{SiC}$ was produced.

When the calcination temperature was raised to $1200{ }^{\circ} \mathrm{C}$, NCD film started to react with the Si substrate, and the phase of SiC could be observed, as indicated by XRD result (in figure 3). As revealed by the XRD spectra, SiC thin film exhibited typical diffractions at $2 \theta$ values of $36^{\circ}, 41^{\circ}, 60^{\circ}, 72^{\circ}$ and $75^{\circ}$, corresponding to the (1 11 1), (2 00 ), (2 200$)$, (3 111 ) and (2 22 2) planes of $\beta$-SiC (JCPDS card no. 29-1129), respectively. The broad morphology of $\mathrm{SiC}$ diffraction peaks results from the nanosized crystals of sample.
The morphology and crystal structure of SiC films synthesized were further studied in detail. The typical SEM images of the SiC film synthesized by HF-PECVD system are shown in figure 4(a). The image shows that

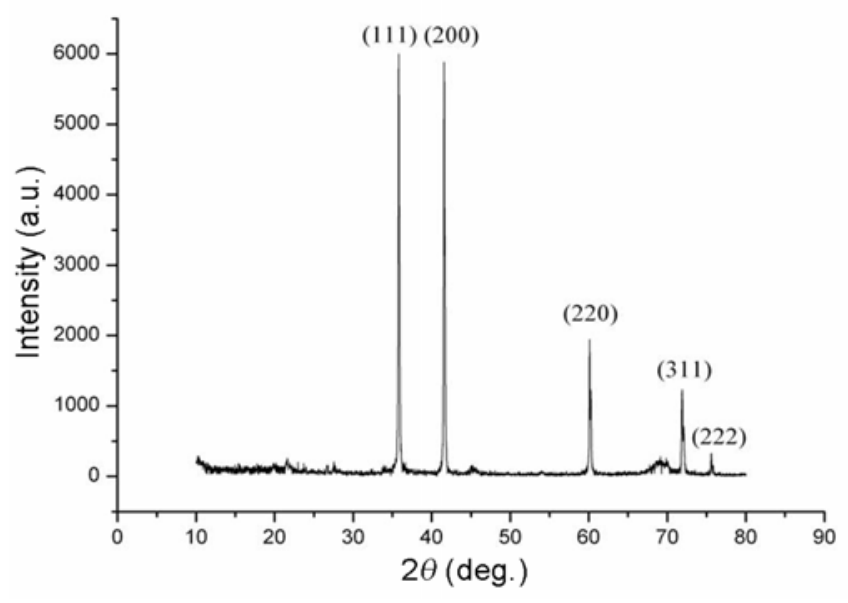

Figure 3. XRD pattern of nc-SiC film.
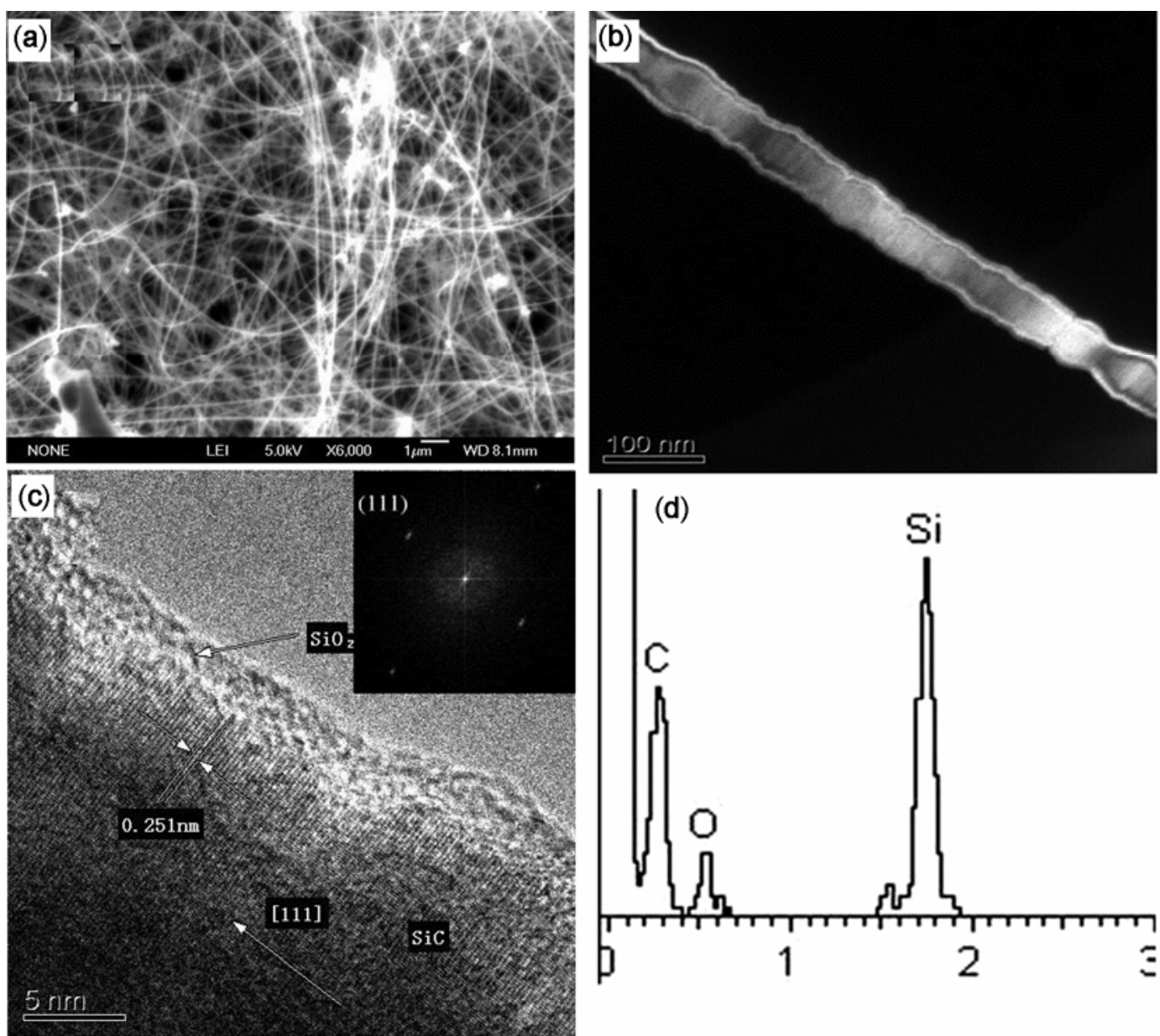

Figure 4. Morphology and EDS spectrum of SiC nanowires. (a) SEM; (b) TEM; (c) HRTEM and (inset is the corresponding fast Fourier transform) and (d) EDS. 


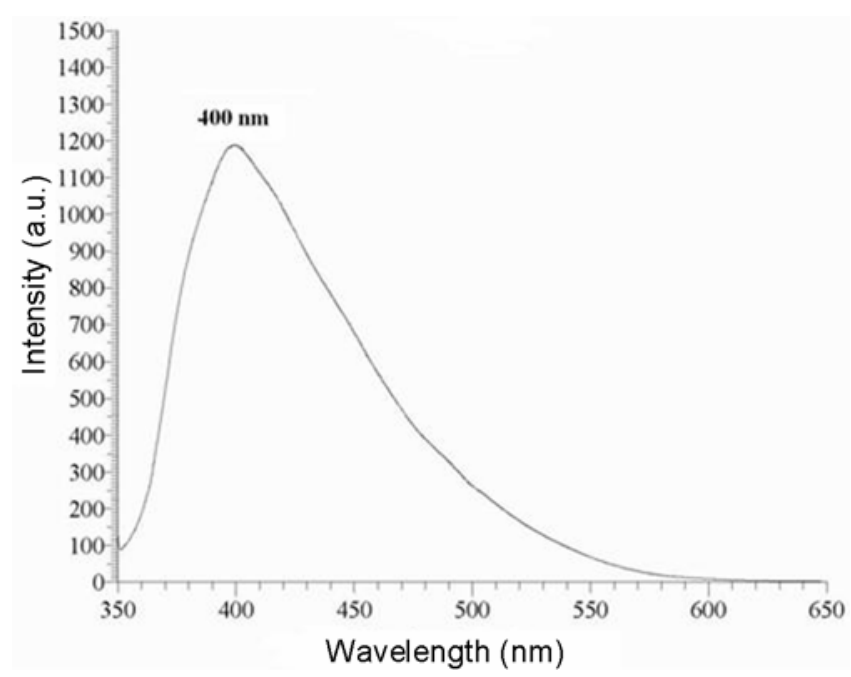

Figure 5. Room temperature PL spectrum of nc-SiC film.

the synthesized films mainly consist of large quantities of randomly oriented $\beta$-SiC nanowires. The nanowires are uniform in diameter of about $50 \mathrm{~nm}$ and lengths up to tens of micrometers. Figure 4(b) displays a typical TEM image of the $\mathrm{SiC}$ nanowires, revealing that the periphery of the $\mathrm{SiC}$ nanowires is very clean and straight. It also shows that the SiC nanowires possess a high density of planar defects, stacking faults which are perpendicular to the wires axes, similar to the already reported results (Pham 2001). With regard to energetic consideration, the formation of stacking faults during the growth of SiC nanowires is favourable due to the contribution of stacking faults themselves with lower energy. By HR-TEM image (figure 4(c)) observation, the nanowires have a crystal core and an amorphous $\mathrm{SiO}_{2}$ sheath with thickness about $2 \mathrm{~nm}$. The $\mathrm{SiO}_{2}$ sheath could be easily removed by etching in hydrofluoric acid (HF). The thickness of the $\mathrm{SiO}_{2}$ sheath could be controlled by changing the etching time (Fang et al 2010). The spacing of lattice fringes is $0.25 \mathrm{~nm}$, corresponding to the $\left\{\begin{array}{lll}1 & 1 & 1\end{array}\right\}$ plane spacing, and also indicates that nanowire grows along the $\left\langle\begin{array}{llll}1 & 1 & 1\end{array}\right\rangle$ direction. The fast Fourier transform (FFT, inset of figure $4(c)$ ) indicates that the nanowires only possess $\left\langle\begin{array}{llll}1 & 1 & 1\end{array}\right\rangle$ crystal orientation. The possible chemical composition of the sample was analysed through the EDS data recorded from several pure nanowires (figure 4d). The presence of peaks demonstrates that the nanowires are composed of $\mathrm{Si}, \mathrm{C}$ and small amount of $\mathrm{O}$. It is found that the molecular ratio of $\mathrm{Si} / \mathrm{C} / \mathrm{O}$ of the nanowires is about $3: 2: 2$, which corresponds well to the standard SiC and $\mathrm{SiO}_{2}$. The small quantity of oxygen may come from the resident oxide layer.

To investigate PL properties of the $\beta$-SiC thin film, the corresponding measurement was carried out at room temperature and a PL spectrum (figure 5) was obtained. When excited with light from a xenon source (excitation wavelength $354 \mathrm{~nm}$ ), the thin film has an emission band between 330 and $600 \mathrm{~nm}$. It is clear that a strong peak centred at $400 \mathrm{~nm}$ is observed. Compared with previously reported luminescence from the bulk of $\mathrm{SiC}$, the emission peak for $\beta$-SiC is nanowires and thin is obviously shifted to the blue. The emergence of the peak with a blue shift is due to the existence of oxygen defects in the amorphous layer, the special rough core-shell interface and the morphology effects such as stacking faults in the nanowires' core (Zhou et al 2000). It also may be attributed to the quantum confinement effect because of the small size.

\section{Conclusions}

In summary, the $\beta$-SiC thin films were synthesized by HF-PECVD system. The as-synthesized thin film mainly consist of $\beta$-SiC nanowires with a diameter of about $50 \mathrm{~nm}$ and tens of micrometers long. By means of XRD, SEM, EDS, PL and TEM (HR-TEM), $\beta$-SiC nanowires have been characterized and discussed in detail. The growth direction of nanowires lies along the $\left\langle\begin{array}{llll}1 & 1 & 1\end{array}\right\rangle$ direction. The tentative growth model according to the $\mathrm{SiC}$ thin film growth process was suggested. Finally, optical property is found in the photoluminescence emission from $\beta$-SiC nanowires, which is different from previous observations of SiC materials. Morphology and size confinement effects are used to interpret the larger blue shift. The SiC nanowires thin film will express excellent in fields of high mechanical strength material, and will be of use for application in electronic circuits, in light-emitting devices and in other advanced block nanodevices.

\section{References}

Borowiak E, Ruemmeli M H, Gemming T, Knupfer M, Biedermann K, Leonhardt A, Pichler T and Kalenczuk R J 2005 J. Appl. Phys. 9756102

Chen J, Wu R, Yang G, Pan Y, Lin J, Wu L and Zhai R 2008 J. Alloys Compd. 456320

Fan J Y, Wu X L and Chu P K 2006 Prog. Mater. Sci. 51983

Fang J, Aharonovich I, Levchenko I, Ostrikov K, Spizzirri P G, Rubanov S and Prawer S 2012 Cryst. Growth Des. 122917

Fernandez S, Kong X, Gotschke T, Calarco R, Geelhaar L, Trampert A and Brandt O 2012 Nano Lett. 126119

Guruvenket S, Andrie S, Simon M, Johnson K W and Sailer R A 2012 ACS Appl. Mater. Interface 45293

Hsu C H, Cloutier S G, Palefsky S and Xu J 2010 Nano Lett. 103272

Huang S M and Hong F C N 2006 Surf. Coat. Technol. 2003160

Huang Y, Wang Q, Wang M, Fei Z and Li M 2012 Rare Met. 31198

Li K Z, Wei J, Li H J, Li Z J, Hou D S and Zhang Y L 2007 Mater. Sci. Eng. A460-461 233 
Lien S Y, Weng K W, Huang J J, Hsu C H, Shen C T, Wang C C, Lin Y S, Wuu D S and Wu D C 2011 Curr. Appl. Phys. 11 S21

Paul W 2008 J. Phys. Chem. C112 12432

Pauschitz A, Koch J S and Eisenmenger C 2000 Bull. Mater. Sci. 26585

Pham C 2001 J. Catal. 200400

Qamar A, Mahmood A, Sarwar T and Ahmed N 2011 Appl. Surf. Sci. 2576923
Vaghri E, Khalaj Z, Ghoranneviss M and Borghei M 2011 J. Fusion Energy 30447

Wang J J N 2007 J. Phys. Chem. B111 4368

Xu J, Yang L, Rui Y, Mei J, Zhang X, Li W, Ma Z, Xu L, Huang X and Chen K 2005 Solid State Commun. 133565

Yu W, Wang X, Geng C, Lve X, Lu W and Fu G 2011 Appl. Surf. Sci. 2581733

Zhou X T, Peng H Y, Shang N G, Wang N, Bello I and Lee C S 2000 Chem. Phys. Lett. 332215 